\title{
Is there a place for ipsilesional eye patching in neglect rehabilitation?
}

\author{
N. Soroker ${ }^{1}$, T. Cohen², C. Baratz ${ }^{2}$, J. Glicksohn² and M. S. Myslobodsky² \\ 'Loewenstein Rehabilitation Hospital, Raanana, and the Sackler School of Medicine, Tel-Aviv \\ University, Tel-Aviv, and ${ }^{2}$ The Psychobiology Research Unit, Tel-Aviv University, Tel-Aviv, \\ Israel
}

\author{
Correspondence to: N. Soroker, Loewenstein Hospital Rehabilitation Center, Department of \\ Stroke Rehabilitation, 278 Ahuza Street, Raanana 43100, Israel
}

\begin{abstract}
Neglect behavior of experimental animals with unilateral posterior cortical lesions improves with the placement of a second lesion in the contralesional superior colliculus or in the intercollicular commissure. Given that the retinotectal fibers are mainly crossed, it has been speculated that ipsilesional eye patching, by depriving the contralesional superior colliculus of its main facilitatory visual input, might achieve similar results, and thus be used as a remediation maneuver in patients with neglect. From six patients with severe persistent neglect, only one showed an unequivocal beneficial effect from ipsilesional eye patching. We discuss the factors which possibly underlie success and failure with this procedure, and the place for it in neglect rehabilitation.
\end{abstract}

Keywords: Hemispatial neglect - Monocular vision - Ocular dominance - Rehabilitation - Stroke

\section{INTRODUCTION}

Experimental animals with large posterior cortical lesions develop disturbances of visual perception and visually guided behavior in the contralateral space, resembling the human hemispatial neglect syndrome. Subsequently the visual loss partially recovers, but if a second lesion is then placed in the superior colliculus ipsilateral to the cortical damage, the animal loses its previously recovered abilities. In contrast, if the second lesion is placed in the contralateral superior colliculus, or in the intercollicular connections, recovery of visual function in the impaired field is facilitated (Sprague, 1966). This well-known effect is usually explained in terms of a homolateral corticotectal system, subserving visuoperceptual and attentional processes related to the contralateral hemispace. Damage to the cortical component of the system on one side causes unevenness of reciprocal inhibition existing between the homological circuits, in favor of the contralesional system. The morphologically intact tectal component of the ipsilesional system can partially compensate for the loss induced by the cortical damage, if it is released from the strong inhibition coming from the other side via the tectal commissures (Kinsbourne, 1987; Rizzolatti and Camarda, 1987).

Based on this logic, Posner and Rafal (1987) have suggested a non-invasive maneuver for the remediation of patients with hemispatial neglect. The retinotectal fibers, unlike the geniculo-striate pathways, are largely crossed (Hendrickson et al., 1970; Hubel et al., 1975; Pollack and Hickey, 1979). Thus, by patching the right eye, one deprives the left superior colliculus of its main facilitatory visual input. Consequently, the inhibition it is capable of exerting on the right superior colliculus will be reduced. It is conceivable that the partially recovered right superior colliculus will then be able to promote orienting toward the left hemispace, with a consequent amelioration of hemineglect. A recent study by Butter and Kirsch (1992), utilizing maneuvers expected to induce collicular activation (monocular patching and lateralized visual stimulation), indicates a potential beneficial effect for such techniques, as could be recognized in some if not all common tests of neglect in their sample (Buttr and Kirsch, 1992).

We examined Posner and Rafal's idea in six patients with severe continual left-sided neglect, using the line-bisection paradigm. Our aim was to assess whether patients suffering from hemispatial neglect are likely to benefit from ipsilesional eye patching. 


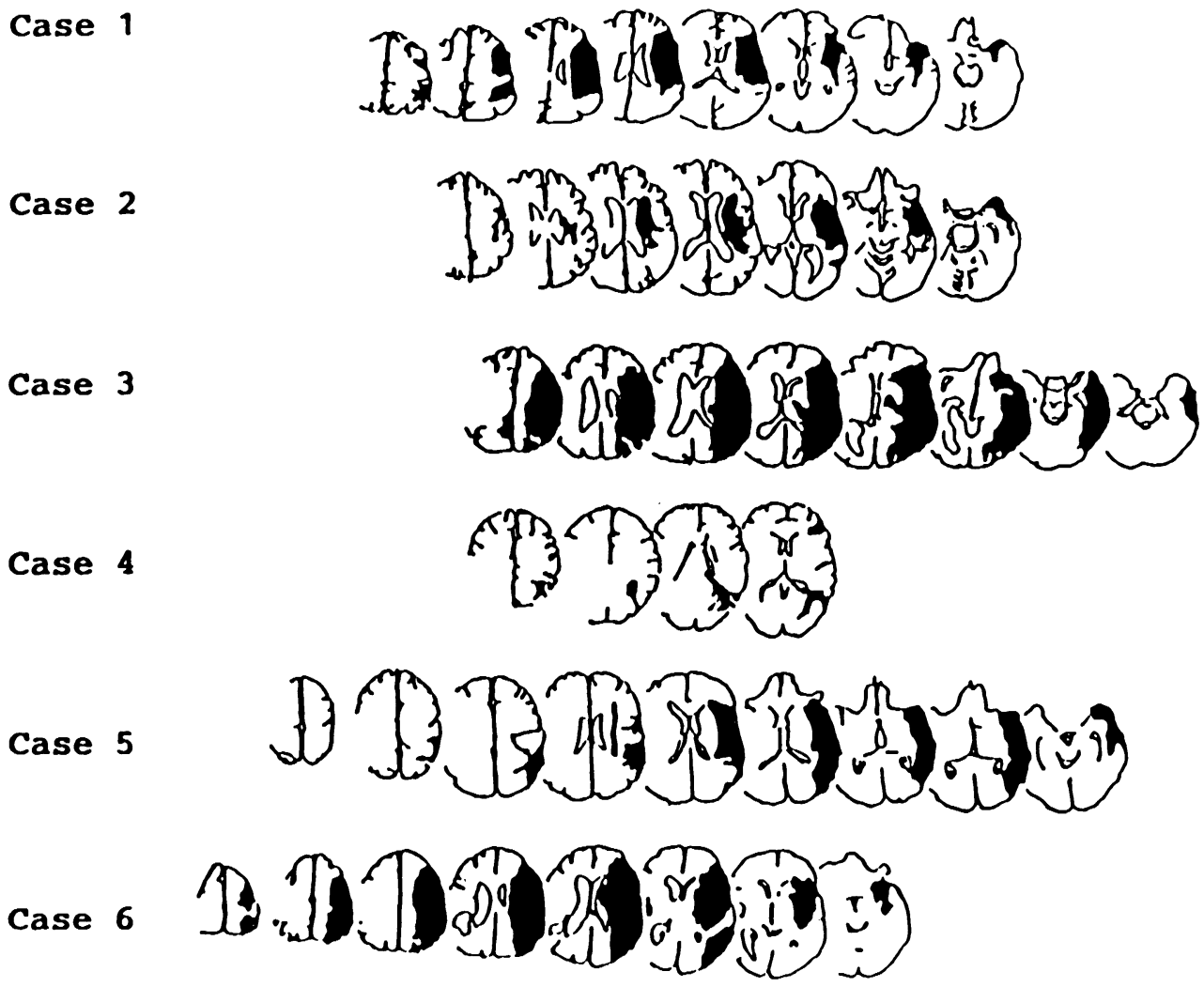

FIG. 1. Reconstructions made from late CT scans of the six patients, demonstrating the location and the extent of the lesions.

\section{METHODS}

\section{Patients}

Six patients admitted to the Loewenstein Hospital for rehabilitation after stroke participated in the study. All of them manifested severe left hemispatial neglect in daily living activities, persisting longer than 2 months after the onset of stroke. The existence of neglect was confirmed using a standardized test battery, the Behavioral Inattention Test (Wilson et al., 1987). There were four males and two females within an age range of 55-64 years (mean $58.7 \pm 3.0$ ). Educational level ranged between 6 and 12 years of schooling (mean $10.3 \pm 2.4$ ). All the patients were right handers. The dominant eye was determined by questioning about eye preference prior to the onset of stroke, in situations demanding monocular vision (like using a camera or a microscope). Also, actual eye preference was evaluated three times in each of the following conditions demanding monocular vision: looking at near and distant objects through a hole, a narrow vertical slit, and a cone opened in its base and apex (the patient's face approaching the base), and holding fixation at the extreme point of ocular vergence. In these situations bias from hand preference and unilateral paralysis is avoided, as no manual activity is demanded. From the results of these tests it was possible to classify two patients as having left ocular dominance, two as having right ocular dominance, and two as having mixed ocular dominance. In all the patients, computed tomography revealed an infarction confined to the territory of the right middle cerebral artery. The right inferior parietal lobule and parts of the right temporal lobe and of the dorsolateral aspect of the right frontal lobe were involved in all the cases (Fig. 1).

This was the first time any neurologic problem had occurred in these patients. (Case 6 is exceptional in having a transient ischemic attack in the past, with right hemiparesis which resolved completely. His CT scan shows a small left-sided subcortical hypodense area, in addition to the right hemispheric infarction.) The patients had a negative history of psychiatric disorders or alcoholism. The study was conducted when the individual patients were 2-8 months after the occurrence of stroke (mean $4.5 \pm 2$ ). At that time the patients were in a stable clinical and metabolic state. They were alert, oriented and cooperative, and clearly understood the task requirements. All the patients had left hemiplegia and hemisensory loss. 
TABLE I. Patients' data

\begin{tabular}{|c|c|c|c|c|c|c|c|c|}
\hline \multirow[t]{2}{*}{ Case } & \multirow[t]{2}{*}{$\begin{array}{l}\text { Age/ } \\
\text { sex }\end{array}$} & \multirow[t]{2}{*}{$\begin{array}{l}\text { Education } \\
\text { (years) }\end{array}$} & \multirow[t]{2}{*}{$\begin{array}{c}\text { Dominant } \\
\text { eye }\end{array}$} & \multirow[t]{2}{*}{$\mathrm{HA}$} & \multicolumn{2}{|c|}{$\begin{array}{l}\text { Corrected } \\
\text { visual acuity }\end{array}$} & \multirow[t]{2}{*}{$\begin{array}{l}\text { TAO } \\
(\mathrm{m})\end{array}$} & \multirow[t]{2}{*}{$\begin{array}{c}\text { BIT } \\
\text { Score }\end{array}$} \\
\hline & & & & & $\mathrm{R}$ & L & & \\
\hline 1 & $55 / \mathrm{M}$ & 12 & L & - & $20 / 100$ & $20 / 50$ & 5.5 & 101 \\
\hline 2 & $57 / F$ & 12 & $\mathrm{~L}$ & - & $20 / 70$ & $20 / 200$ & 8 & 103 \\
\hline 3 & $57 / \mathrm{M}$ & 12 & $\mathrm{R}$ & + & $20 / 30$ & $20 / 30$ & 2 & 69 \\
\hline 4 & $64 / F$ & 8 & $\mathrm{R}$ & + & $20 / 70$ & $20 / 40$ & 4 & 53 \\
\hline 5 & $58 / \mathrm{M}$ & 6 & M & + & $20 / 200$ & $20 / 200$ & 5 & 60 \\
\hline 6 & $61 / \mathrm{M}$ & 12 & $M$ & - & $20 / 40$ & $20 / 40$ & 2.5 & 71 \\
\hline
\end{tabular}

TAO, time after onset (months); BIT, Behavioral Inattention Test (cut-off score for normality = 130); HA, hemianopsia.

TABLE II. Multiple regression analysis: statistics

\begin{tabular}{lrrrrrr}
\hline Case & \multicolumn{5}{c}{ Independent variable } \\
\cline { 2 - 7 } & Line & $\mathrm{d} 1$ & $\mathrm{~d} 2$ & $\begin{array}{c}\mathrm{d} 1 \times \\
\text { line }\end{array}$ & $\begin{array}{c}\mathrm{d} 2 \times \\
\text { line }\end{array}$ & Constant \\
\hline 1 & $1.20^{\star}$ & 1.11 & 0.57 & 0.25 & $-0.70^{\star}$ & $-2.87^{*}$ \\
2 & $1.57^{\star}$ & 0.49 & 0.17 & -0.00 & -0.03 & $-2.87^{*}$ \\
3 & $3.53^{*}$ & 0.62 & 2.05 & $1.70^{*}$ & $2.08^{*}$ & $-10.77^{*}$ \\
4 & 0.11 & -2.85 & $-5.19^{*}$ & $1.12^{*}$ & $1.26^{*}$ & 1.19 \\
5 & $2.35^{*}$ & 0.29 & -0.97 & $2.37^{*}$ & -0.45 & $-4.86^{*}$ \\
6 & $1.44^{*}$ & 2.07 & 2.65 & $-1.56^{*}$ & 0.04 & $-4.64^{*}$ \\
\hline
\end{tabular}

$\mathrm{d} 1=1$, if viewing condition $=$ MR; 0 otherwise. $\mathrm{d} 2=1$, if viewing condition $=M L ; 0$ otherwise.

${ }^{\star} p<0.05$, or better, two-tailed.

Three patients were unable, at the time when the experiments were conducted, to perceive the examiner's fingers in the left hemifield at confrontation, neither in a static position nor with finger movements. These three patients are regarded hemianoptic, although the possibility exists that imperception resulted from neglect. The other three patients manifested extinction of left-sided stimuli upon bilateral simultaneous stimulation. Individual demographic and clinical data are shown in Table I.

\section{Stimuli and procedure}

Patients' performance in a multi-length (to control for possible length-dependent effects) line bisection task was examined in three different conditions: binocular vision (B), monocular vision with the left eye (ML), and monocular vision with the right eye (MR). Testing took place on three consecutive days. The task was similar in essence to the general procedure employed in recent experiments by Marshall and Halligan (1990). Black lines, $1 \mathrm{~mm}$ wide, of 10 different lengths $(18,36,54,72,90,108,126,144,162$ and $180 \mathrm{~mm}$ ), each drawn in the center of a separate sheet of white A4 paper $(208 \times 298 \mathrm{~mm})$, served as stimuli. Each stimulus length was presented 10 times in pseudo-random order. Thus, in each of the three viewing conditions the patient had to bisect a total of 100 lines. The patient was seated in front of a desk and the stimuli were presented one at a time, so that the midpoint of the line coincided with the patient's midsagittal plane. The instructions were to mark the exact midpoint of the line with a fine pen held in the dominant right hand. No time restriction was imposed but corrections were not permitted. The patients were free to make head and eye movements as they wished, but the sitting position in relation to the desk, and the place of the stimulus sheet on the desk top, had to remain fixed. The displacement of the subjective midpoint from the true midpoint was measured with an accuracy of $1 \mathrm{~mm}$. Rightwards displacements were signed positive whereas leftwards displacements received the negative sign.

\section{RESULTS}

Mean signed displacement from the objective midpoint served as the dependent variable in a withinsubject multiple regression analysis (Lorch and Myers, 1990) employing line length, viewing condition [dummy-coded, using two dummy variables $(0 / 1)$ to represent the three values- $-B, M L$, and MR, and the interaction of the latter with line length (i.e. each dummy variable multiplied by line length). Separate analyses were conducted for each patient, and the results of these are reported in Table II.

Figure 2 presents the individual lines for each viewing condition, computed from the data apearing in Table II.

As can be seen from Table II, all regression equations are significant $(\mathrm{F}$ values, with $\mathrm{df}=5.24$, being: $51.00,65.51,144.51,11.48,153.88,21,20$, respectively, all at $p<0.0001)$. Respective $R^{2}$ statistics are $0.91,0.93,0.97,0.71,0.97,0.82$. The interaction of 

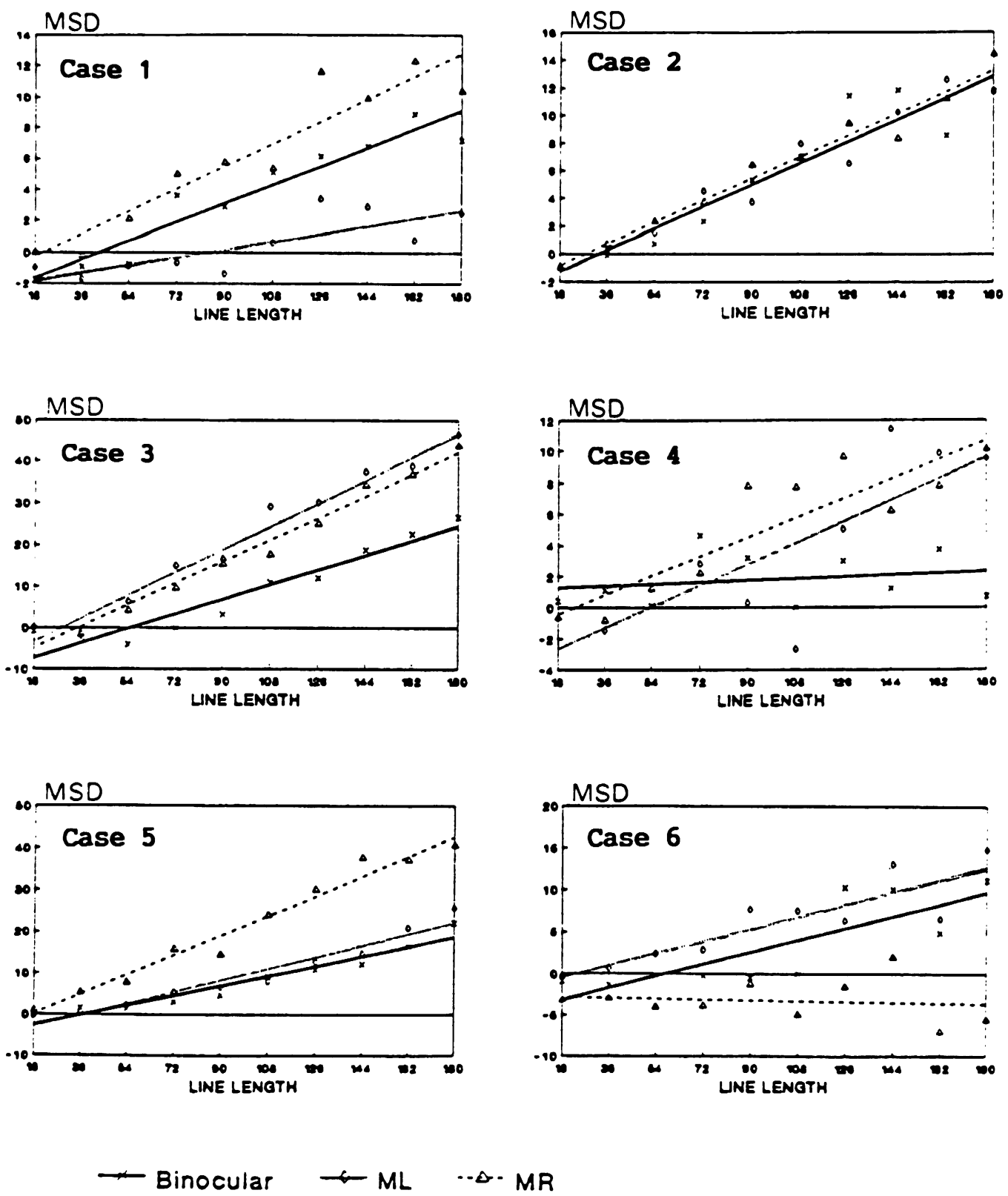

FIG. 2. Mean signed displacement (MSD, in $\mathrm{mm}$ ), as a function of line length (in $\mathrm{mm}$ ). Individual regression lines for each viewing condition are represented separately for each of the six patients.

viewing condition and line length is significant for all subjects, except Case 2.

The prediction by Posner and Rafal (1987) of improvement in hemineglect (a shorter degree of displacement from the true midpoint in the line bisection paradigm) in $\mathrm{ML}$ viewing, is operationalized as a negative regression coefficient for the $\mathrm{d} 2 \times$ line interaction. We note such a significant coefficient only for Case 1 (see also Fig. 2). As a corollary of the PosnerRafal prediction, a greater degree of displacement in MR viewing is operationalized as a positive regres- sion coefficient for the $\mathrm{d} 1 \times$ line interaction. We note such a significant coefficient for Cases 3,4 and 5 (see also Fig. 2).

\section{DISCUSSION}

Posner and Rafal (1987) proposed to use ipsilesional eye patching in cognitive training programs for neglect patients. This novel remedial approach has a sound theoretical basis, as explained in the Introduction. Posner and Rafal did not provide experimental 
data on the effectiveness of their proposed maneuver. However, Butter and Kirsch (1992), who recently examined the effect of monocular patching and lateralized visual stimulation on neglect, were able to show benefits for these maneuvers in at least some common tests for neglect in their sample. In the present study we examined the effect of monocular sighting upon the magnitude of lateralized inattention in a group of six patients with severe left hemineglect. The line bisection paradigm was used to estimate neglect severity in each viewing condition.

The maneuver of ipsilesional eye patching reduced the magnitude of neglect only in one of the six patients examined (see Table II and Fig. 2). In discussing this result both biological and methodological factors should be considered. Posner and Rafal's idea is based essentially on Sprague's (1966) famous animal experiments. However, the ability of mesencephalic visual and attentional systems to compensate for deficits induced by posterior cortical damages, which was demonstrated by Sprague in the cat, might be significantly lower in humans (Milner, 1987). This is related to the different degree of encephalization of functions and to the obvious differences in the role of visuospatial and attentional functions in the two species (Milner, 1987). Also, it is possible that those aspects of recovery from neglect that are mediated by collicular activity, relate mainly to involuntary (bottom-up, stimulus-driven) aspects of spatial attention and orientating, as opposed to voluntary (topdown, data-driven) attentional processes (Butter, 1987). In that case, line bisection performance might not be the optimal measure for detection of colliculus-dependent amelioration in the neglect syndrome.

One of the six patients demonstrated significantly less hemineglect in conditions of left monocular viewing, as compared to the binocular state, raising the possibility that a given subgroup of neglect patients might be more likely to benefit from monocular input than other subgroups. Recruitment of patients for the study was planned with the intention of minimizing interpersonal variance with regard to basic demographic, clinical and lesion data. Thus, the study group was composed of subjects who share in common: right handedness; same age group; same etiology (ischemic infarction, first event); lesion confined to the same vascular territory (right middle cerebral artery); much similar clinical presentation of left-sided hemiplegia and hemi-hypoesthesia; and a state of chronic left hemineglect. The patients varied however in their dominant eye, a factor which seemed to influence the results. As can be seen in Table II, significant amelioration of line bisection performance under left monocular viewing condition occurred only in Case 1 with left ocular dominance. It is possible that the other patient with left ocular dominance, Case 2, could not benefit from left monocular viewing because of her poor visual acuity in the left eye. The mechanism of collicular activation proposed by Posner and Rafal (1987) predicts a significant aggravation of left hemineglect in the right monocular viewing condition. A significant such effect was shown in the present study by the two patients with right ocular dominance (Cases 3 and 4) and by Case 5 with mixed ocular dominance. In general, left and right monocular viewing produced significant but asymmetric effects, and none of the six patients demonstrated both neglect amelioration in left monocular vision and aggravation in right monocular vision. It is hard to explain these results in terms of Posner and Rafal's mechanism of reciprocal inter-collicular inhibition affected by crossed retinotectal facilitation, unless interaction with other factors is taken into account. We suggest that ocular dominance might be an important such factor. The likelihood of amelioration of left hemineglect under left monocular viewing condition, as well as the likelihood of neglect aggravation in right monocular viewing (both expected by Posner and Rafal's mechanism), seem to increase when the eye viewing monocularly is the dominant eye. The exact neurophysiological significance of ocular dominance is not yet clear. However, sighting dominance is known to affect egocentric localization, as well as other perceptual and attentional tasks, and there is some evidence suggesting that ocular dominance may reflect a mechanism whereby the visual inut to the dominant eye receives attentional priority (Mefferd and Wieland, 1969; Mefferd et al., 1969; Porac and Coren, 1976, 1986; Coren and Porac, 1976, 1982; Seyal et al., 1981). The small number of patients in the present study precludes definite conclusions in this regard. However, it seems that future studies of the effect of monocular sighting in neglect should look for the possible confounding effect of ocular dominance.

As far as can be judged from this preliminary study, Posner and Rafal's maneuver bears a potential beneficial effect for only a minority of neglect patients. Moreover, since the procedure actually restricts the visual information from the attended right visual field, it might even increase the disability already existing. As many neglect patients recover either spontaneously or with the help of conventional treatment modalities, it is felt that prior to implementation of monocular input as a remediation technique, the factors underlying success and failure with this procedure should be thoroughly investigated and 
recognized. Apart from the possible effect of ocular dominance and the effects of different demographic and clinical characteristics of the patients, stimulus effects should be controlled, as suggested also by the recent findings of Butter and Kirsch (1992). Paradigms other than line bisection might prove to be more suitable for the purpose of detecting important aspects of spatial behavior, which are mediated by brainstem structures.

\section{REFERENCES}

Butter CM (1987) Varieties of attention and disturbances of attention: a neuropsychologial analysis. In: Neurophysiological and Neuropsychological Aspects of Spatial Neglect (Ed. M. Jeannerod), pp. 1-23. Elsevier, Amsterdam.

Butter CM and Kirsch N (1992) Combined and separate effects of eye patching and visual stimulation on unilateral neglect following stroke. Archives of Physical Medicine and Rehabilitation, 73, 1133-1139.

Coren S and Porac C (1976) Size accentuation in the dominant eye. Nature, 260, 527-528.

Coren S and Porac C (1982) Monocular asymmetries in visual latency as a function of sighting dominance. American Journal of Optometry and Physiological Optics, 59, 987-990.

Hendrickson A, Wilson ME and Toyne MJ (1970) The distribution of optic nerve fibers in Macaca mulata. Brain Research (Amsterdam), 23, 425-427.

Hubel DH, Levay S and Wiesel TN (1975) Mode of termination of retinotectal fibers in macaque monkey: an autoradiographic study. Brain Research (Amsterdam), 96, 25-40.

Kinsbourne M (1987) Mechanisms of unilateral neglect. In: Neurophysiological and Neuropsychological Aspects of SpatialNeglect(Ed. M. Jeannerod), pp.69-86. Elsevier,Amsterdam.

Lorch RF and Myers JL (1990) Regression analyses of repeated measures data in cognitive research. Journal of Experimental Psychology: Learning, Memory, and Cognition, 16, 149-157.
Marshall JC and Halligan PW (1990) Line bisection in a case of visual neglect: psychophysical studies with implications for theory. Cognitive Neuropsychology, 7, 107130.

Mefferd RB and Wieland BA (1969) Influence of the eye dominance on the apparent centers of simple horizontal lines. Perceptual and Motor Skills, 28, 847-850.

Mefferd RB, Wieland BA and Dufilho PL (1969) Systematic alterations of the apparent centers of lines. Perceptual and Motor Skills, 28, 803-825.

Milner AD (1987) Animal models for the syndrome of spatial neglect. In: Neurophysiological and Neuropsychological Aspects of Spatial Neglect (Ed. M Jeannerod), pp. 259-288. Elsevier, Amsterdam.

Pollack JG and Hickey TL (1979) The distribution of retino-collicular axon terminals in rhesus monkey. Journal of Comparative Neurology, 185, 587-602.

Porac C and Coren S (1976) The dominant eye. Psychological Bulletin, 83, 880-897.

Porac C and Coren S (1986) Sighting dominance and egocentric localization. Vision Research, 26, 1709-1713.

Posner MI and Rafal RD (1987) Cognitive theories of attention and the rehabilitation of attentional deficits. In: Neuropsychological Rehabilitation (Eds MJ Meir, A Benton and L Diller), pp. 182-201. Guilford Press, New York.

Rizzolatti G and Camarda R (1987) Neural circuits for spatial attention and unilateral neglect. In: Neurophysiological and Neuropsychological Aspects of Spatial Neglect (Ed. M Jeannerod), pp. 289-313. Elsevier, Amsterdam.

Seyal M, Sato S, White BG and Porter RJ (1981) Visual evoked potential and eye dominance. Electroencephalography and Clinical Neurophysiology, 52, 424-428.

Sprague JM (1966) Interaction of cortex and superior colliculus in remediation of visually guided behavior in the cat. Science, 123, 1544-1546.

Wilson B, Cockburn J and Halligan PW (1987) Behavioral Inattention Test. Thames Valley Test Company, Titchfield, Hants.

(Received 12 July 1994; accepted as revised 27 October 1994) 


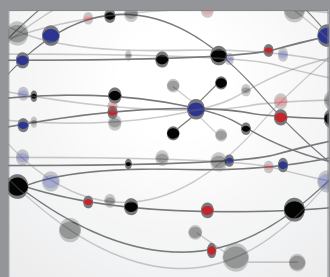

The Scientific World Journal
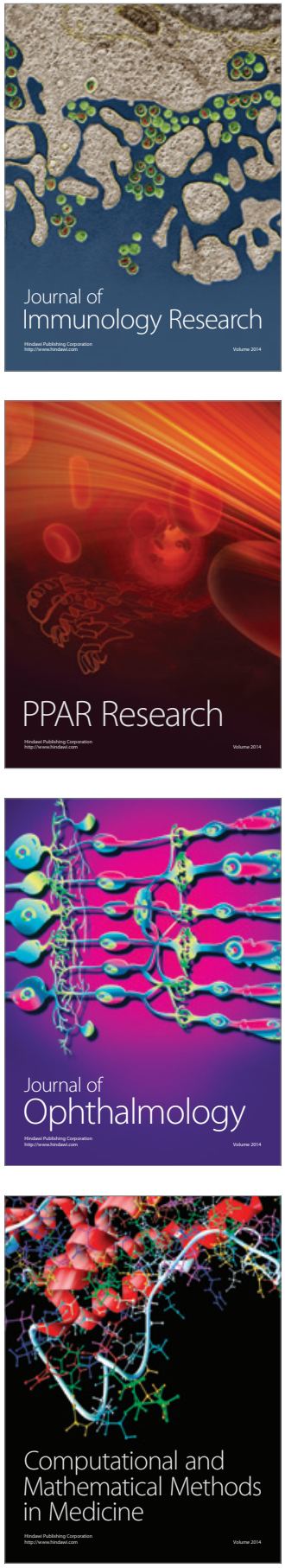

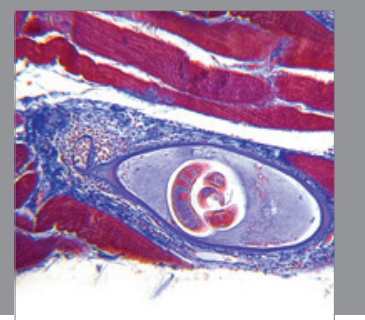

Gastroenterology

Research and Practice
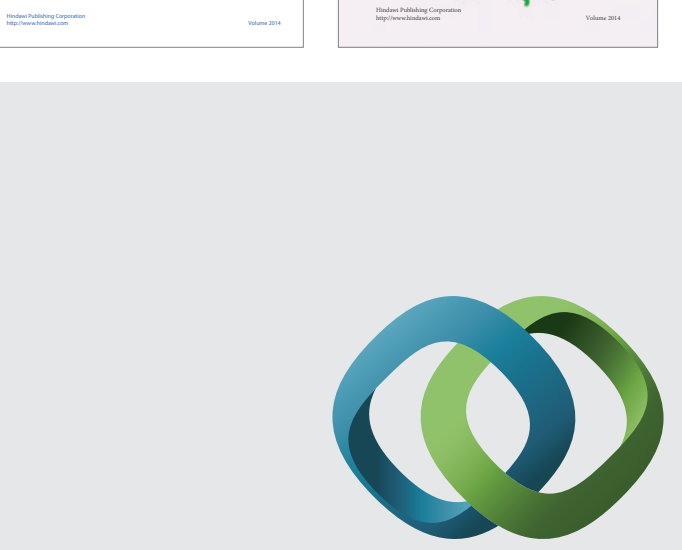

\section{Hindawi}

Submit your manuscripts at

http://www.hindawi.com
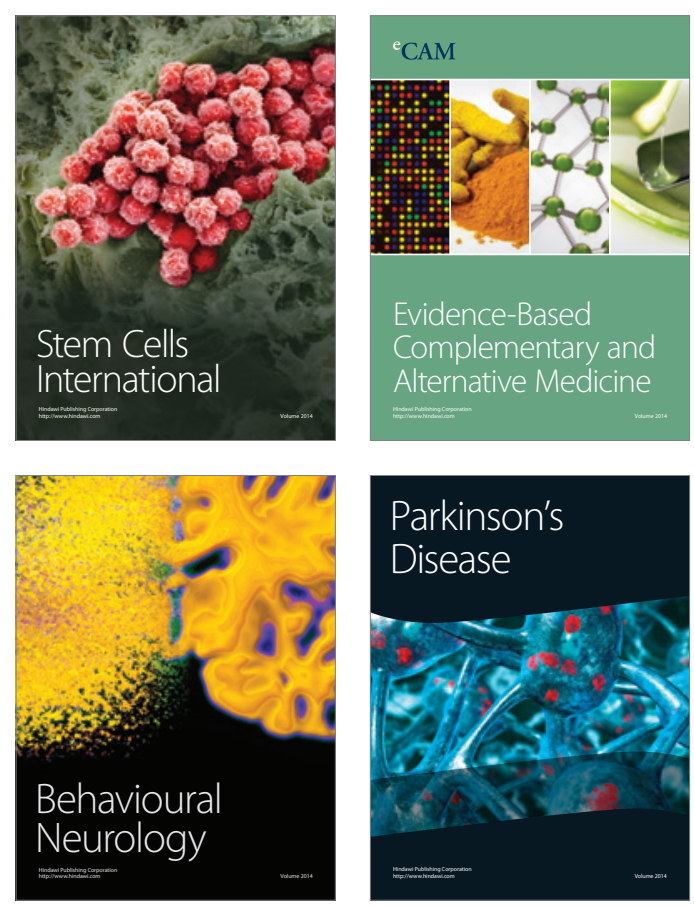

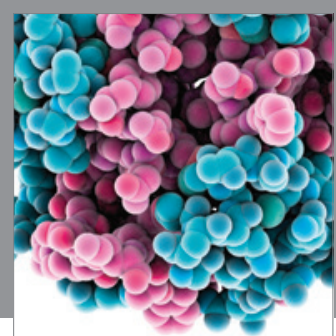

Journal of
Diabetes Research

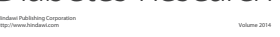

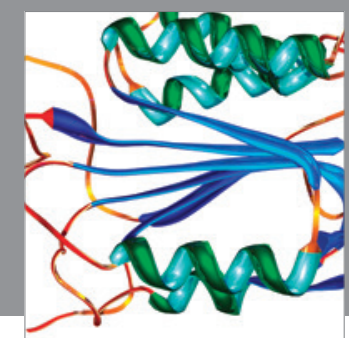

Disease Markers
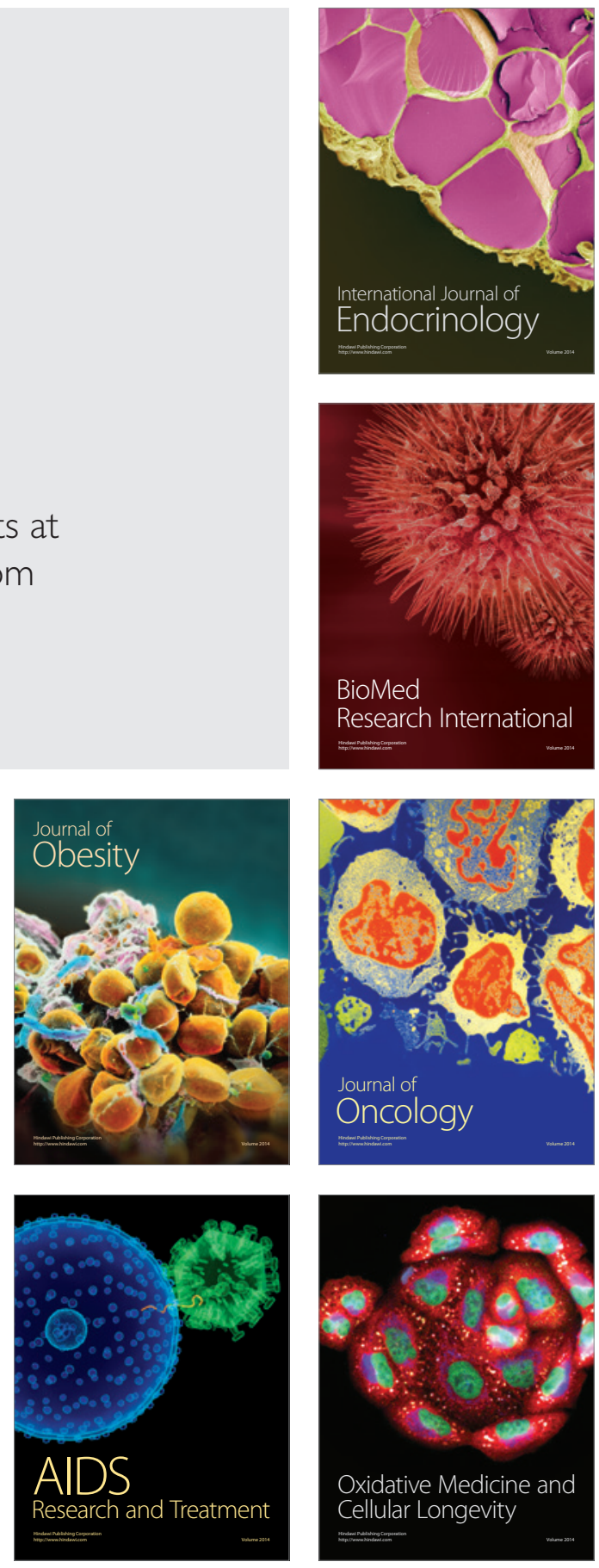